\title{
MarkerMouse: Mouse Cursor Control Using a Head-Mounted Marker
}

\author{
Rados Javanovic and Ian Scott MacKenzie \\ Dept. of Computer Science and Engineering \\ York Univeristy \\ Toronto Ontario Canada M3J 1P3 \\ \{rados, mack\} @cse. yorku.ca
}

\begin{abstract}
We propose MarkerMouse, an inexpensive method for controlling the mouse cursor using a web cam and a marker placed on the user's forehead. Two modes of cursor control were compared: position-control and velocitycontrol. In position-control mode the cursor is positioned where the user's head is pointing. In velocity-control mode the mouse cursor moves in a constant speed in the direction the user's head is pointing. In an experiment designed according to ISO 9241-9, we found a mean throughput $1.61 \mathrm{bps}$ in positioncontrol mode. Throughput was $34 \%$ less, or $1.07 \mathrm{bps}$, in velocity-control mode. We explain how from the marker image we control the mouse cursor position and reduce noise in our computations.
\end{abstract}

Keywords: User interfaces, cursor control, web cam, marker tracking, head position tracking, head-operated mouse, mouse emulation, ISO 9241-9.

\section{Introduction}

Interacting with desktop computers typically engages both hands of the user. This is not a problem for most people, but for people with certain disabilities this scenario may be untenable. Fortunately, there is considerable research on providing efficient access to computers for disabled users. Several interesting technologies and solutions have emerged such as blink-based text entry [7] and voice-based mouse cursor control [4].

People with severe disabilities who cannot use, or have limited use of, their hands and who cannot speak might not be able to control an on-screen cursor. Sometimes the only option is to use the motion of the head to control the cursor. However, commercial solutions are typically expensive. Origin Instruments' HeadMouse Extreme, for example, costs about one thousand U.S. dollars (http://www.orin.com). A solution that costs less would be highly desirable. A new method for head cursor control is proposed in this paper.

Our proposed method does not require expensive hardware. It uses a common web camera, a printout of a marker (Fig. 1a) mounted on the user's forehead, and the software to process the marker orientation and move the cursor. Notable, as well, is that the user-to-computer interface is wireless, making it comfortable to use. We refer to this head-mounted marker system for mouse cursor control as MarkerMouse. 

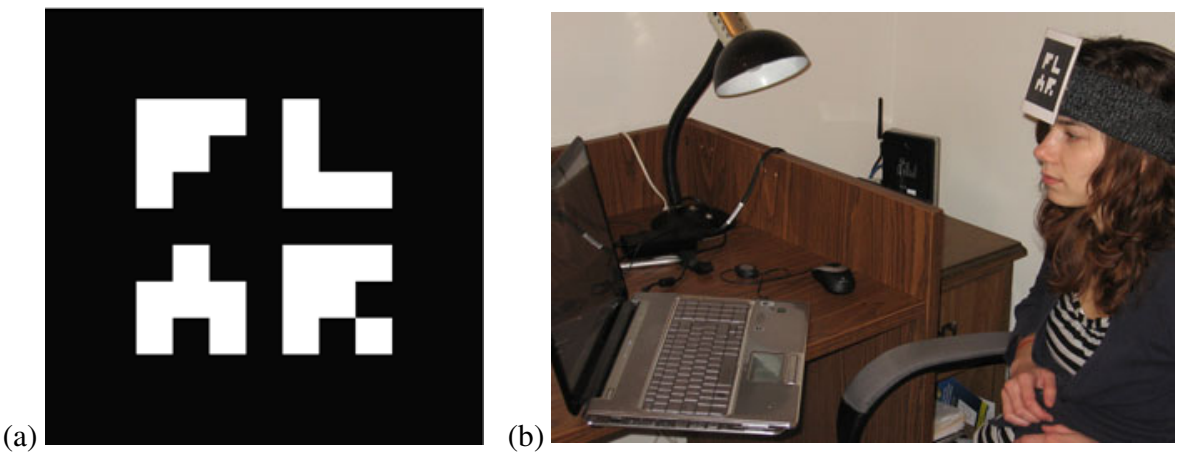

Fig. 1. MarkerMouse (a) printout (b) participant using MarkerMouse

The system is intended for people with restricted motor-control in the arms since only head motion is required to move the mouse cursor. The rest of this paper is organized as follows. In the next section, we review related work. Following this, we describe our system and provide details on the interface and noise reduction algorithm. Finally, we present an evaluation of our system, designed according to the ISO 9241-9 standard for evaluating non-keyboard input devices [5].

\section{Related Work}

Most uses of head-mounted markers are in virtual worlds to connect the physical position and movements of a real person with the virtual objects they interact with. For example, Knoeflein et al. [6] used a head-mounted marker in a virtual world gaming application. An IR marker was attached to a head-mounted camera and tracked to determine the user's head pose while playing ping-pong. Applications for cursor control tend to use face and feature tracking, rather than makers $[2,11]$. In these applications, the focus is on the algorithms rather than the interaction. The use of a head-mounted marker greatly simplifies the software, since the geometric features in the marker are both precise and user-independent.

\section{MarkerMouse}

In this section, we describe the MarkerMouse interface, the position-control and velocity-control modes of cursor control, button clicks, and noise reduction.

\subsection{Interface Details}

The MarkerMouse head-mounted marker system is simple to assemble. The user simply prints the marker, positions it in some manner on her forehead, installs the software, and performs a simple calibration procedure. The marker can be attached to the user's glasses, a hat, a bandana, or anything that might be comfortable. 
For MarkerMouse to work, the user must sit in front of a web camera so that the marker on the user's forehead is clearly visible (Fig. 1b). The orientation of the marker with respect to the camera when the user is looking straight at the monitor is the "neutral orientation". The neutral orientation is sampled and used in computing the movement of the marker and, hence, the motion of the mouse cursor.

\subsection{Image Processing}

The software linking the marker orientation to mouse cursor movement processes the marker image as input by the web camera. The first task is to compute the marker's normal vector in 3D space. Since the marker has a distinct, non-symmetric pattern on a square background it is possible to take an image captured by the web camera and retrieve the $3 \mathrm{D}$ plane on which the marker is sitting. By finding this plane, we calculate the normal vector of the marker. This is the neutral orientation. When the marker undergoes movement, we take the angle between the new normal vector and the neutral orientation and use this to determine where to move the cursor. To compute the marker's normal vector we used a software library called NyARToolkit ${ }^{1}$.

\subsection{Button Clicks}

Our primary focus is on emulating mouse cursor control, apart from the functionality of clicking. To emulate a mouse button click, our system uses any key press on the system's keyboard. For accessible computing, depending on the disability, users may have enough manual facility to press a switch, for example using their hand. For users with severe disabilities who are not able to use their arms, the mouse button clicks can be emulated in different ways, for example, using blinks [7, 10], intentional muscle contractions [1, 3] or a sip-and-puff straw [12].

\section{Position-Control and Velocity-Control}

We evaluated two different modes of cursor control: position-control and velocitycontrol. In position-control mode, the marker orientation on the user's head determines the final position of the cursor on the screen. For example, if the user looks straight at the monitor the cursor will be in the center of the screen. If the user looks left the cursor will be on the left side of the screen, and if he then looks straight ahead again the cursor will be in the center again. This is similar to the operation of a stylus on a tablet or a finger on a touchscreen.

In velocity-control mode, the head orientation determines the direction to move the cursor. If the user looks straight at the monitor, the cursor is motionless. If the user looks left the cursor starts moving left and continues moving left while the user continues to look left. Movement is initiated when the computed displacement is equivalent to 200 pixels of mouse displacement. The rate of cursor movement is constant at 150 pixels per second. On the test system, this amounts to $10 \%$ horizontally and $16 \%$ vertically of the display extent per second. In velocity-control mode, we constrained the mouse movement to eight directions: up, down, left, right, and along the four diagonals.

\footnotetext{
${ }^{1}$ Available from Virtual Worldlets (http://www.virtualworldlets.net/).
} 


\subsection{Noise Reduction}

Having the mouse cursor position directly dependent on the orientation of the marker may cause the cursor to jump around due to camera noise and inaccuracies in computing the marker's transformation. We used two methods to minimize noise.

The first method uses dampening. Instead of using the marker's normal vector to position the cursor directly, we compute the normal vector as a tentative cursor position. For each step the cursor moves accordingly, but with added dampening, as follows. Before the cursor is moved, a vector is computed in the direction of movement. The length of the vector is the distance between the two positions. This vector is multiplied by a dampening constant, $D(0<D<1)$, and then the cursor takes a step accordingly:

$$
\text { NewMousePos }=\text { MousePos }+(\text { TargetPos }- \text { MousePos }) \times D
$$

In our application, we set $D$ to 0.5 . For a large head movement, the cursor moves relatively fast. For a small head movement, the cursor moves somewhat more slowly. This helps reduce jitter in the cursor movement due to small variations in the computation of the marker's normal vector.

The second method uses a history of the mouse cursor position. Previous cursor positions are recorded and used in computing the new position. Each new cursor position was determined using the computed, new position averaged with the last 10 mouse positions. This sliding-average approach helps minimize big spikes in the change of the marker's normal vector due to noise.

Applying these two methods together, the mouse cursor behaviour is more stable.

\section{Method}

While the design and initial testing of MarkerMouse system were promising, a proper evaluation with a pool of participants is required to validate the design. Our experimental evaluation tested how well participants could execute point-select tasks with our MarkerMouse in each mode of cursor control. The evaluation also included a generic mouse as a baseline condition.

Participants. Nine able-bodied participants were recruited from the local university campus. Five were male, four female, Ages ranged from 22 to 27 years. All participants used a computer on a regular basis.

Apparatus (Hardware and Software). Participants worked on a Hewlett Packard Pavilion $d v 7$ laptop computer. The system includes an embedded web camera on the top of the display. The resolution of the display was $1440 \times 900$ pixels. The operating system was Microsoft Vista (Home Edition).

Two separate software utilities were used. For demonstrating MarkerMouse and to provide participants the opportunity to practice, a simple program presenting an array of circular targets was created. See Fig. 2a. The targets were of random diameters in the range 30-80 pixels and were randomly scattered about the display. The targets could be selected in any order. When a target was clicked on, the colour changed to green to give visual feedback of a successful selection. 
(a)

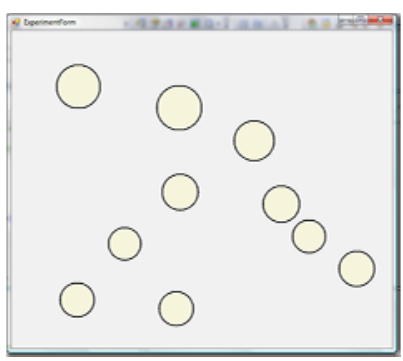

(b)

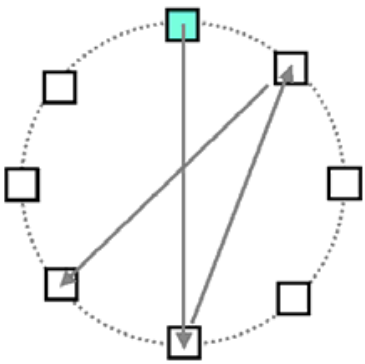

Fig. 2. Software (a) practice (b) ISO 9241-9 task \#2

The experiment software was an implementation of task \#2 in ISO 9241-9. See Fig. 2b. Eight square targets were uniformly positioned around a layout circle with a diameter of 600 pixels. Each target was $80 \times 80$ pixels. The participant first clicked on the top target, then on the target directly opposite, then on the target next to the first target, and so on around the layout circle. At any time, the target to select was highlighted. The target colour changed during selection. Selecting all targets was a "sequence". This was repeated five times per mode of control.

Procedure. Participants sat in front of the laptop computer. The marker was put on the participant's forehead clearly visible to the web-cam. The participant was asked to look straight into the screen and with a press of a button the current orientation of the marker was saved. This orientation was the neutral orientation and was used for system calibration. The calibration was done only once before each condition. Before each condition, participants spent 10 minutes getting familiar with the head-mounted marker using the practice software. For each condition, the participant was instructed on how the mode of control worked and on what was expected. Testing started after any button was pressed.

After the completion of all tests a questionnaire was handed to the participants to solicit their impressions on the input modes. The questionnaire was based on response items in ISO 9241-9 [5]. Each item had a rating from 1 to 5, as follows:

Smoothness during operation (1: very smooth -5 : very rough)

Mental effort required for operation 1: too low - 5: too high)

Accurate pointing (1: easy - 5: difficult)

Neck fatigue (1: none -5 : very high)

General comfort (1: very uncomfortable -5 : very comfortable)

Overall the input device was (1: very difficult to use -5 : very easy to use)

Experiment design. The experiment was a $3 \times 5$ within-subjects design, with the following independent variables and levels:

Mode of control: Position-control, Velocity-control, Generic Mouse

Sequence: $\quad 1,2,3,4,5$

Participants were divided into three groups with the mode of control conditions administered using a Latin square. 
The experiment included throughput as the dependent variable, as specified in ISO 9241-9. Throughput, in bits per second (bps), is a composite measure combining the speed and accuracy of participant responses. After each sequence of trials, throughput was calculated using

$$
\text { Throughput }=I D_{e} / M T
$$

$M T$ is the mean movement time per trial over all trials in a sequence. $I D_{e}$ is the effective index of difficulty, computed as

$$
I D_{e}=\log _{2}\left(D / W_{e}+1\right)
$$

where $D$ is the distance in pixels between the two targets (600 pixels) and $W_{e}$ is the width of the distribution of clicks. $W_{e}$, is computed using

$$
W_{e}=4.133 \times S D_{x}
$$

where $S D_{x}$ is the standard deviation in selection coordinates along the axis of approach. Details are provided in other papers [e.g., 9].

\section{Results and Discussion}

Throughput. As clearly seen in Fig. 3a, there was a significant effect of mode of control on throughput $\left(F_{3,8}=555.7, p<.0001\right)$. Not surprisingly, the mouse performed best. Its throughput of $4.42 \mathrm{bps}$ is consistent with mouse results in other ISO 9241-9 evaluations [9], thus validating our methodology.

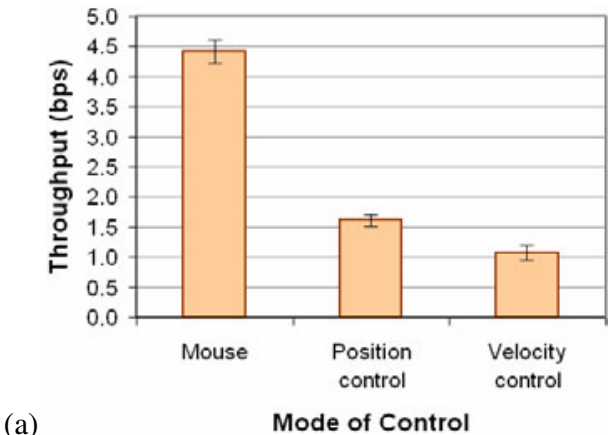

(a)

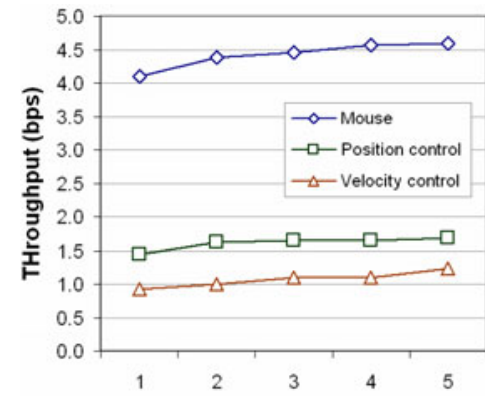

(b)

Sequence

Fig. 3. Throughput (a) by mode of control (b) by sequence

The mean throughput for position-control movement of 1.61 bps was about $34 \%$ higher than the throughput of $1.07 \mathrm{bps}$ observed for velocity-control movement. While substantially less than the throughput for the mouse, these figures are consistent with throughput for other pointing devices, such as a joystick (1.8 bps; see [8]).

As the trials continued, the throughput for both the position-control and velocitycontrol modes improved (Fig. 3b). After the first sequence, the position-control mode did not improve much, but the throughput for the velocity-control mode kept increasing. 
Position-control mode produced better results because the mouse cursor went to the target position determined by the marker orientation almost instantaneously. In velocity-control mode, the mouse cursor had a constant speed (150 pixels/second). This slowed velocity-control mode, as multiple iterations of move and adjust were needed to position the cursor on the target.

Participant feedback. As MarkerMouse is intended for accessible computing and testing was done with able-bodied participants, there was an expected bias favoring the mouse. In comparing the two modes of control for MarkerMouse, participants indicated an overall preference for the position-control mode, rating it better than velocity-control mode for both neck fatigue and accurate pointing. Participants, however, gave slightly better scores to velocity-control mode for mental effort and smoothness.

\section{Conclusions}

In this paper we introduced a new method of controlling a mouse cursor using a marker placed on the user's forehead. Two methods of cursor control, position-control and velocity-control, were compared along with a desktop mouse. The mean throughputs for the position- and velocity-control modes were $1.61 \mathrm{bps}$ and $1.07 \mathrm{bps}$, respectively, compared to the desktop mouse mean throughput of $4.42 \mathrm{bps}$.

Noise in marker detection was a limitation for the position-control mode. Velocitycontrol mode was less influenced by noise. However, because velocity-control is less intuitive, velocity-control mode performed worse than position-control mode.

An improvement for position-control movement would be a better way to deal with the marker detection noise, to improve precision and reduce jitter. This problem can be approached from two directions: to have a more robust method for marker detection and marker orientation calculation, and to have a better smoothing algorithm that minimizes noise after the orientation was computed.

\section{References}

1. Barreto, A.B., Scargle, S.D., Adjouadi, M.: A practical EMG-based human-computer interface for users with motor disabilities. Journal of Rehabilitation Research 37, 53 (2000)

2. Chauhan, V., Morris, T.: Face and feature tracking for cursor control. In: Proceedings of the Scandinavian Conference on Image Analysis (Unpublished, 2001); retrieved from http: / / www. cs.man.ac.uk/ tmorris/SCIA630.pdf (December 23, 2009)

3. Felzer, T., Nordmann, R., Rinderknecht, S.: Scanning-based human-computer interaction using intentional muscle contractions. In: Smith, M.J., Salvendy, G. (eds.) Universal Access in Human-Computer Interaction, Part II, HCII 2009. LNCS, vol. 5618, pp. 309-318. Springer, Heidelberg (2009)

4. Harada, S., Landay, J.A., Malkin, J., Li, X., Bilmes, J.A.: The vocal joystick: Evaluation of voice-based cursor control techniques. In: Proceedings of the ACM Conference on Computers and Accessibility - ACCESS 2006, pp. 187-204. ACM, New York (2006)

5. ISO, Ergonomic requirements for office work with visual display terminals (VDTs) - Part 9: Requirements for non-keyboard input devices (ISO 9241-9), International Organisation for Standardisation. Report Number ISO/TC 159/SC4/WG3 N147, February 15 (2000) 
6. Knoerlein, B., Szekely, G., Harders, M.: Visuo-haptic collaborative augmented reality ping-pong. In: Proceedings of the International Conference on Advances in Computer Entertainment Technology - ACE 2007, pp. 91-94. ACM, New York (2007)

7. MacKenzie, I.S., Ashtiani, B.: BlinkWrite: Efficient text entry using eye blinks. In: Universal Access in the Information Society (UAIS) (in press)

8. MacKenzie, I.S., Kauppinen, T., Silfverberg, M.: Accuracy measures for evaluating computer pointing devices. In: Proceedings of the ACM Conference on Human Factors in Computing Systems - CHI 2001, pp. 119-126. ACM, New York (2001)

9. Soukoreff, R.W., MacKenzie, I.S.: Towards a standard for pointing device evaluation: Perspectives on 27 years of Fitts' law research in HCI. International Journal of HumanComputer Studies 61, 751-789 (2004)

10. Su, N.M., Mark, G.: Communication chains and multitasking. In: Proceedings of the ACM Conference on Human Factors in Computing Systems - CHI 2008, pp. 83-92. ACM, New York (2008)

11. Tu, J., Tao, H., Huang, T.: Face as mouse through visual face tracking. Computer Vision and Image Understanding 108, 35-40 (2007)

12. Yanco, H.A.: Wheelesley: A robotic wheelchair system: Indoor navigation and user interface. In: Mittal, V.O., Yanco, H.A., Aronis, J., Simpson, R. (eds.) Assistive Technology and Artificial Intelligence, pp. 256-268. Springer, Berlin (1998) 